
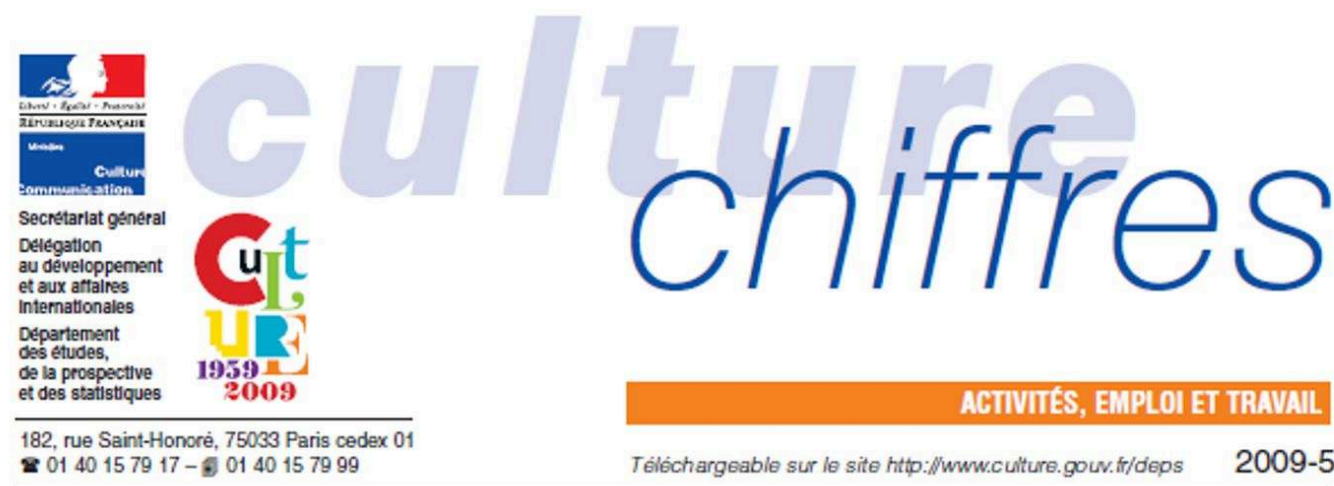

\title{
L'insertion professionnelle des diplômés des établissements supérieurs de la culture
}

Éric Cléron*

Professional integration of graduates from cultural higher education establishments

Environ 5500 personnes ont obtenu en 2004 un diplôme d'un établissement supérieur artistique et culturel relevant du ministère de la Culture et de la Communication $^{1}$ : $50 \%$ sont diplômés en arts plastiques, $27 \%$ en architecture, $18 \%$ sont diplômés du spectacle (musique, danse, théâtre, spectacles et cinéma) et $5 \%$ dans le domaine du patrimoine et des musées. Il s'agit de diplômes d'écoles supérieures et non d'université, de niveau bac +3 ou plus.

En complément de l'enquête Génération ${ }^{2}$ qu'il mène à intervalle régulier depuis 1992, le Centre d'études et de recherches sur les qualifications (Cereq) a étudié, à la demande du ministère de la Culture et de la Communication, l'insertion professionnelle des diplômés d'établissements d'enseignement supérieur artistique et culturel relevant du ministère de la Culture et de la Communication ${ }^{3}$ au cours des trois premières années sur le marché du travail : délai d'obtention du premier emploi, rapport entre celui-ci et la qualification, situation d'emploi trois ans après l'obtention du diplôme, et, enfin, événements survenus en cours d'insertion.

Les résultats montrent que les diplômes de la culture sanctionnent, d'une façon générale, des formations professionnalisantes : le premier emploi est obtenu rapidement (un an ou moins) et il est très souvent en rapport avec la formation et la qualification des diplômés. Trois ans plus tard, le constat est confirmé et l'emploi est souvent stabilisé. Selon les filières, les résultats varient toutefois: si les diplômés de l'architecture s'insèrent mieux sur le marché de l'emploi, l'accès à un emploi stable est moins aisé pour les diplômés des arts plastiques.

* Attaché statisticien à l'Insee.

1. Voir la définition du champ de l'enquête, page 10.

2. Voir la description des enquêtes Génération du Cereq, pase 10

3. Dans la suite du document, les diplómés d'un établissement d'enseignement supérieur relevant du ministère de la Culture et de la Communication sont appelés, par commodité, les diplômés de la culture. 


\section{L'insertion professionnelle des diplômés des établissements supérieurs de la culture}

Professional integration of graduates from cultural higher education establishments

\section{Eric Cléron}

Éditeur : Département des études, de la prospective et des statistiques

Lieu d'édition : Paris

Année d'édition : 2009

Date de mise en ligne : 21 septembre 2015

Collection : Culture chiffres

ISBN électronique : 9782111398467

\section{Sobooks}

http://books.openedition.org

\section{Édition imprimée}

Date de publication : 1 juillet 2009

Nombre de pages : 12

\section{Référence électronique}

CLÉRON, Eric. L'insertion professionnelle des diplômés des établissements supérieurs de la culture.

Nouvelle édition [en ligne]. Paris : Département des études, de la prospective et des statistiques, 2009

(généré le 25 avril 2021). Disponible sur Internet : <http://books.openedition.org/deps/575>. ISBN

9782111398467. 


\title{
L'insertion professionnelle des diplômés des établissements supérieurs de la culture
}

\author{
Éric Cléron*
}

\author{
Professional integration of graduates \\ from cultural higher education establishments
}

Environ 5500 personnes ont obtenu en 2004 un diplôme d'un établissement supérieur artistique et culturel relevant du ministère de la Culture et de la Communication ${ }^{1}: 50 \%$ sont diplômés en arts plastiques, $27 \%$ en architecture, $18 \%$ sont diplômés du spectacle (musique, danse, théâtre, spectacles et cinéma) et $5 \%$ dans le domaine du patrimoine et des musées. Il s'agit de diplômes d'écoles supérieures et non d'université, de niveau bac +3 ou plus.

En complément de l'enquête Génération ${ }^{2}$ qu'il mène à intervalle régulier depuis 1992, le Centre d'études et de recherches sur les qualifications (Cereq) a étudié, à la demande du ministère de la Culture et de la Communication, l'insertion professionnelle des diplômés d'établissements d'enseignement supérieur artistique et culturel relevant du ministère de la Culture et de la Communication ${ }^{3}$ au cours des trois premières années sur le marché du travail : délai d'obtention du premier emploi, rapport entre celui-ci et la qualification, situation d'emploi trois ans après l'obtention du diplôme, et, enfin, événements survenus en cours d'insertion.

Les résultats montrent que les diplômes de la culture sanctionnent, d'une façon générale, des formations professionnalisantes : le premier emploi est obtenu rapidement (un an ou moins) et il est très souvent en rapport avec la formation et la qualification des diplômés. Trois ans plus tard, le constat est confirmé et l'emploi est souvent stabilisé. Selon les filières, les résultats varient toutefois : si les diplômés de l'architecture s'insèrent mieux sur le marché de l'emploi, l'accès à un emploi stable est moins aisé pour les diplômés des arts plastiques.

\footnotetext{
* Attaché statisticien à l'Insee.

1. Voir la définition du champ de l'enquête, page 10.

2. Voir la description des enquêtes Génération du Cereq, page 10.

3. Dans la suite du document, les diplômés d'un établissement d'enseignement supérieur relevant du ministère de la Culture et de la Communication sont appelés, par commodité, les diplômés de la culture.
} 


\section{DES FILIÈRES PROFESSIONNALISANTES À COURT TERME}

\section{Un accès rapide au premier emploi}

La génération 2004 des diplômés de la culture a fait ses premiers pas dans la vie active dans une conjoncture peu porteuse. Entre 2004 et 2006, le chômage a légèrement augmenté de $8,7 \%$ à 9,2\% pour ensuite diminuer jusqu'à $7,3 \%$ de la population active. Presque tous ont obtenu un emploi au cours de leurs trois premières années sur le marché du travail : seuls $6 \%$ n'ont jamais travaillé au cours de cette période, et parmi ces $6 \%$, environ un individu sur trois n'a pas connu de période de chômage, étant principalement en inactivité. L'obtention de ce premier emploi a été rapide : $90 \%$ l'ont trouvé au cours de l'année qui a suivi l'obtention de leur diplôme (graphique 1). Le temps d'accès médian à ce premier emploi est de 2 mois.

\section{Graphique 1 - Temps d'accès au premier emploi des diplômés de la culture en 2004}

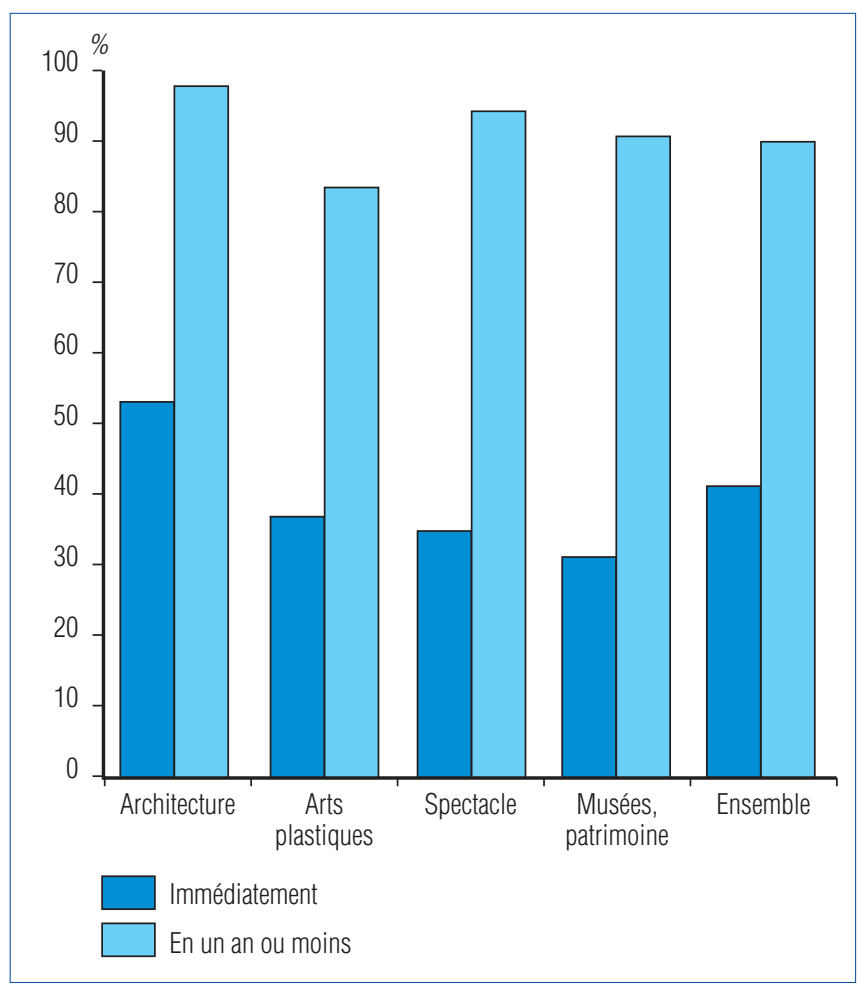

Source : Cereq/DEPS, Enquête génération 2004, ministère de la Culture, 2009
Quelques différences apparaissent suivant le domaine de formation: si $98 \%$ des diplômés en architecture ayant eu un emploi l'ont obtenu en un an ou moins, ce n'est le cas que pour $84 \%$ des diplômés des arts plastiques.

\section{Un premier emploi en lien ${ }^{4}$ avec la formation reçue}

Quatre diplômés sur cinq ont trouvé leur premier emploi dans le domaine de leur diplôme (tableau 1). la situation varie cependant selon la filière. Ainsi, plus de neuf diplômés sur dix de l'architecture ou du spectacle ont commencé par travailler dans leur domaine, alors que c'est le cas pour environ sept diplômés sur dix des arts plastiques ou des musées et patrimoine. Pour autant, une partie des emplois exercés hors du champ d'application du diplôme se situe cependant dans le champ culturel. C'est notamment le cas pour les diplômés des arts plastiques, dont une partie d'entre eux trouve un premier emploi dans le spectacle vivant.

\section{Tableau 1 - Domaine du premier emploi des diplômés de la culture en 2004}

\begin{tabular}{|lccc|}
\hline Filière & En lien & Sans lien & Ensemble \\
Architecture & 95 & 5 & 100 \\
Spectacle & 93 & 7 & 100 \\
Musées, patrimoine & 73 & 27 & 100 \\
Arts plastiques & 67 & 33 & 100 \\
Ensemble & $\mathbf{8 0}$ & $\mathbf{2 0}$ & $\mathbf{1 0 0}$ \\
& & & \\
Note de lecture : pour 95\% des diplômés de l'architecture, le premier emploi \\
est en rapport avec leur formation. \\
\hline \multicolumn{4}{c}{ Source : Cereq/DEPs, Enquête génération 2004, } \\
& ministère de la Culture et de la Communication, 2009
\end{tabular}

\section{Correspondance entre le niveau du diplôme} et la qualification du premier emploi occupé

La comparaison entre le niveau de formation requis pour l'emploi occupé et le niveau de diplôme est l'un des indicateurs du lien entre diplôme et emploi. $78 \%$ des emplois occupés sont d'un niveau au moins égal à profession intermédiaire (tableau 2). D'une façon générale, le déclassement ${ }^{5}$ est assez faible. De plus, les emplois concernés par

\footnotetext{
4. Un emploi est en lien avec le diplôme si le domaine d'activité de l'emploi correspond à la formation reçue. Ainsi, un diplômé d'une école d'architecture étant assistant d'architecte a un emploi en lien avec la formation reçue. À l'inverse, un diplômé d'une école d'architecture devenu éclairagiste a un emploi qui n'est pas en lien avec la formation reçue.

5. Le déclassement désigne ici un niveau de formation requis pour l'emploi inférieur au niveau de formation du salarié. La table de correspondance entre le diplôme et la catégorie socioprofessionnelle a permis d'établir cette comparaison. Voir Emmanuelle NAUZE-FICHET et Magda ToMASINI, «Diplôme et insertions sur le marché du travail : approche socioprofessionnelle et salariale du déclassement », Économie et statistiques, no 354 , avril 2002. Il faut toutefois souligner que d'autres formes de déclassement existent, comme par exemple le rapport entre niveau de diplôme et condition salariale de l'emploi.
} 


\begin{tabular}{|c|c|c|c|c|c|}
\hline $\begin{array}{r}\text { Chefs } \\
\text { et profession }\end{array}$ & $\begin{array}{l}\text { ntreprise, cadres } \\
\text { tellectuelles supérieures }\end{array}$ & $\begin{array}{c}\text { Professions } \\
\text { intermédiaires }\end{array}$ & Employés & Ouvriers & Ensemble \\
\hline Architecture & 47 & 50 & 2 & 1 & 100 \\
\hline Arts plastiques & 16 & 53 & 20 & 11 & 100 \\
\hline \multicolumn{6}{|l|}{ Spectacle } \\
\hline Diplôme de niveau bac +3 & 79 & 15 & 4 & 2 & 100 \\
\hline Diplôme de niveau bac +2 & 73 & 21 & - & 6 & 100 \\
\hline Musées, patrimoine & 17 & 41 & 40 & 2 & \\
\hline Tout domaine & 34 & 44 & 17 & 5 & 100 \\
\hline
\end{tabular}

Source : Cereq/DEPS, Enquête génération 2004, ministère de la Culture et de la Communication, 2009

le déclassement sont essentiellement des emplois sans lien avec le diplôme. Ainsi, seul environ un emploi déclassé sur cinq est un emploi en lien avec le diplôme.

Les diplômés de la filière spectacle semblent protégés du déclassement dans le cadre de leur premier emploi : respectivement $86 \%$ et $94 \%$, selon le niveau de diplôme, ont un emploi dont la qualification correspond à leur niveau de diplôme. C'est le cas pour $59 \%$ seulement des diplômés des arts plastiques, $58 \%$ pour ceux des musées et patrimoine. Enfin, on observe que la moitié des diplômés de l'architecture commence avec un emploi de niveau profession intermédiaire (pour la plupart, des postes d'assistant d'architecte).

\section{$85 \%$ des diplômés de la culture occupent un emploi en rapport avec leur formation trois ans plus tard}

L'observation sur la correspondance entre domaine et niveau de formation et emploi est confirmée trois ans plus tard. Plus de huit diplômés sur dix ont un emploi en rapport avec leur formation à la date de l'enquête. Quelle que soit la filière, ils sont au moins les trois quarts à être dans ce cas (tableau 3).

Par rapport au premier emploi obtenu après leur entrée sur le marché du travail, on observe une augmentation importante (+ 10 points) de l'emploi en lien avec le diplôme pour les diplômés des arts plastiques. Parmi les diplômés ayant un emploi sans lien avec le diplôme, une partie travaille dans le secteur culturel, notamment dans le spectacle vivant, où ils sont éclairagistes ou monteurs audiovisuels par exemple.
Tableau 3 - Domaine de l'emploi à la date de l'enquête

\begin{tabular}{|lcc|}
\hline & En lien & Sans lien \\
Architecture & 94 & 6 \\
Spectacle & 93 & 9 \\
Arts plastiques & 76 & 34 \\
Musées, patrimoine & 72 & 28 \\
Ensemble & $\mathbf{8 4}$ & 16 \\
& & \\
Note de lecture : $94 \%$ des diplômés de l'architecture ayant un emploi à la \\
date de l'enquête ont un emploi en rapport avec leur formation.
\end{tabular}


Amplitude et PÉRENNité

DE L'EMPLOI

\section{SELON LES FILIÈRES ARTISTIQUES}

\section{Le premier emploi est de longue durée lorsqu'il correspond à la filière de formation}

La moitié des diplômés de la culture a eu un premier emploi d'une durée de 18 mois ou plus (tableau 4), soit environ $50 \%$ du temps total (les trois années au cours desquelles l'insertion est étudiée). La durée d'emploi est directement corrélée à la correspondance de l'emploi avec la filière de formation : dans le cas d'un emploi en rapport avec le diplôme, la durée médiane d'occupation de cet emploi est de 24 mois, alors qu'elle n'est plus que de 9 mois dans le cas d'un emploi sans rapport avec la formation. Cela montre la probable volonté de s'insérer dans un emploi en rapport avec le domaine de formation, les diplômés restant, à l'inverse, peu de temps dans des emplois sans lien avec leur diplôme.

La durée moyenne du premier emploi varie cependant fortement suivant la filière : de 24 mois

Tableau 4-Durée du premier emploi

\begin{tabular}{|lcc|}
\hline \multirow{2}{*}{$\begin{array}{l}\text { Emploi en rapport } \\
\text { avec la formation }\end{array}$} & \multicolumn{2}{c|}{ Durée } \\
\cline { 2 - 3 } Oui & moyenne & médiane \\
Non & 22 & 24 \\
Ensemble & 13 & 9 \\
& 19 & 18 \\
Note de lecture : la durée moyenne du premier emploi est de 19 mois. \\
\hline \multicolumn{2}{r}{ Source : Cereq/DEPs, Enquête génération 2004, } \\
& ministère de la Culture et de la Communication, 2009
\end{tabular}

pour les diplômés du spectacle à 14 mois pour ceux des musées et patrimoines (tableau 5). Enfin, on observe que $43 \%$ des diplômés occupent toujours ce premier emploi à la date de l'enquête, soit trois ans après leur entrée sur le marché de l'emploi.

\section{Plus de la moitié de CDD pour}

\section{le premier emploi quelle que soit la filière}

Plus de la moitié des diplômés sont embauchés pour une durée déterminée (contrat en CDD) pour leur premier emploi (tableau 6), et ceci quelle que soit la filière. C'est cependant beaucoup moins que l'ensemble de leur génération : $69 \%$ des diplômés en 2004 toutes filières confondues ont commencé en CDD, et $19 \%$ en intérim. Seul un diplômé sur cinq a obtenu un contrat à durée indéterminé pour son premier emploi (que cet emploi soit en lien avec le diplôme ou non).

On observe l'importance de l'indépendance (16\% de l'ensemble), en particulier dans les filières des arts plastiques et de l'architecture : les diplômés des arts plastiques, avec plus de tra-

\section{Tableau 5 - Durée du premier emploi selon la filière}

\begin{tabular}{|c|c|c|c|c|}
\hline \multirow{3}{*}{$\begin{array}{l}\text { Domaine } \\
\text { du diplôme }\end{array}$} & \multicolumn{2}{|c|}{ Durée } & \multicolumn{2}{|c|}{ Emploi actuel } \\
\hline & \multicolumn{2}{|c|}{$\begin{array}{c}\text { moyenne médiane } \\
\text { (en mois) }\end{array}$} & \multicolumn{2}{|c|}{$\begin{array}{l}\text { oui } \\
(\text { (en } \%)\end{array}$} \\
\hline & 21 & 19 & 41 & 59 \\
\hline Spectacle & 26 & 24 & 65 & 35 \\
\hline Arts plastiques & 17 & 13 & 37 & 63 \\
\hline Musées, patrimoine & e 14 & 6 & 38 & 62 \\
\hline Ensemble & 20 & 18 & 43 & 57 \\
\hline \multicolumn{5}{|c|}{$\begin{array}{l}\text { Note de lecture : le premier emploi des diplômés de l'architecture a duré e } \\
\text { moyenne } 21 \text { mois. }\end{array}$} \\
\hline
\end{tabular}

Tableau 6 - Statut d'emploi du premier contrat

\begin{tabular}{|c|c|c|c|c|c|c|}
\hline \multirow[b]{2}{*}{ Filière } & \multicolumn{5}{|c|}{ Statut } & \multirow[t]{2}{*}{ Ensemble } \\
\hline & CDD & CDI & Contrats aidés & $\begin{array}{l}\text { Indépendants } \\
\text { (à leur compte) }\end{array}$ & Autres & \\
\hline Architecture & 56 & 27 & 1 & 14 & 2 & 100 \\
\hline Arts plastiques & 49 & 19 & 5 & 22 & 5 & 100 \\
\hline Spectacle & 68 & 22 & - & 5 & 5 & 100 \\
\hline Musées, patrimoine & 52 & 29 & - & 5 & 5 & 100 \\
\hline Ensemble & 55 & 22 & 2 & 16 & 5 & 100 \\
\hline
\end{tabular}


vailleurs indépendants que de CDI (respectivement $22 \%$ et $19 \%$ ) et les diplômés de l'architecture avec $14 \%$ dans ce cas.

La part de contrats à durée déterminée pour un premier emploi est particulièrement importante parmi les diplômés du spectacle, ce qui peut s'expliquer par l'importance de l'intermittence parmi les emplois dans le secteur du spectacle. Ces contrats à durée déterminée sont cependant de durée assez longue, plus de la moitié d'entre eux durant 31 mois ou plus à la date de l'enquête (il s'agit de cas de CDD reconduits, comptés cependant comme étant encore le premier emploi).

À l'inverse, le CDI n'est pas pour autant gage de durée. Ainsi, parmi les diplômés, $20 \%$ ont obtenu leur premier emploi dans le cadre d'un CDI mais ont depuis quitté cet emploi. Parmi ces individus, on observe que $38 \%$ avaient un emploi sans lien direct avec leur diplôme contre $20 \%$ de ceux pour qui ce premier CDI est encore leur emploi à la date de l'enquête. Malgré tout, le fait de quitter un emploi en CDI n'est pas rédhibitoire quant à la suite de l'insertion. En effet, $85 \%$ de ces individus sont en situation d'emploi à la date de l'enquête, contre $9 \%$ au chômage.

\section{Des emplois à temps complet pour la filière architecture, donc des revenus plus importants}

Pour les deux tiers des diplômés, le premier emploi est un emploi à temps complet (tableau 7), soit une proportion inférieure à celle de l'ensemble des diplômés de la génération 2004 (80 \% commencent par un emploi à temps complet). Ce sont les diplômés du spectacle qui sont le plus touchés par le temps partiel (57\% d'entre eux). Là encore, l'importance de l'intermittence dans ce secteur explique pour partie ce phénomène. Cette forte proportion des emplois occupés à temps partiel est l'une des principales caractéristiques des diplômés de la culture.

Conséquence du nombre de diplômés employés à temps complet, la moitié des diplômés de l'architecture ont débuté leur premier emploi avec un salaire mensuel net supérieur à 1500 euros, alors que pour les autres filières le salaire médian se situe entre 1000 et 1100 euros. Globalement, le salaire médian des diplômés de la culture dans leur premier emploi est plus élevé de 100 euros que pour l'ensemble de la génération 2004.

Lorsqu'on retire l'effet du temps $\operatorname{partiel}^{6}$, le salaire net des diplômés du spectacle augmente fortement, passant d'un salaire médian de 1058 euros à 1300 euros, et celui des diplômés des arts plastiques, où l'on trouve une forte proportion d'indépendants, de 1074 euros à 1200 euros.

Ainsi, les diplômés de l'architecture connaissent une insertion plus rapide et plus durable que les autres diplômés de la culture, même si pour eux aussi, le recours au contrat à durée déterminée comme premier contrat d'embauche prédomine.

Tableau 7 - Temps de travail du premier emploi et revenu

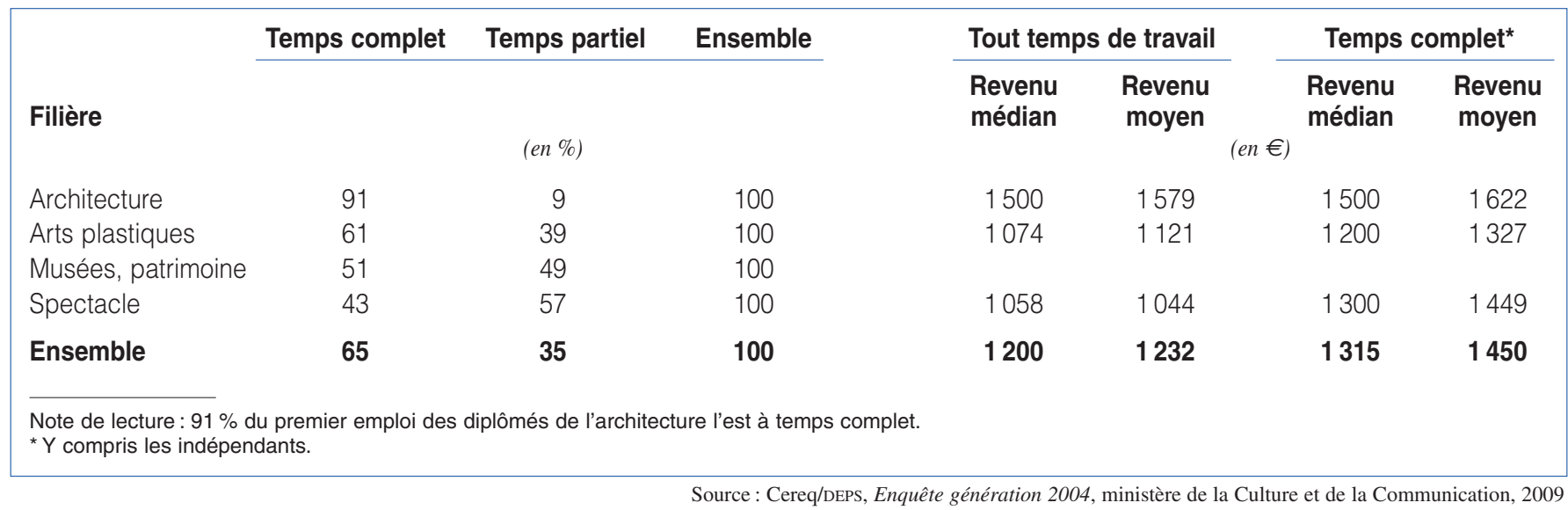

6. Les travailleurs indépendants sont considérés comme travaillant à temps complet. 
QUALITÉ DE L'EMPLOI TROIS ANS APRÈS L'OBTENTION DU DIPLÔME

Trois ans après l'obtention de leur diplôme, quatre titulaires sur cinq d'un diplôme de la culture ont un emploi

$81 \%$ des diplômés de la culture ont un emploi à la date de l'enquête, trois années après l'obtention de leur diplôme (tableau 8), une proportion supérieure à la moyenne de l'ensemble de la génération 2004 (77 \%), et de l'ordre de l'ensemble des diplômés de niveau bac +3 ou 4 . Pour près de la moitié des diplômés (49\%), cet emploi à la date de l'enquête est toujours le premier emploi, en particulier pour les diplômés du spectacle qui sont deux sur trois à occuper, trois plus tard, leur premier emploi.

\section{Un taux de chômage variable selon les filières}

Le taux de chômage est de $12 \%$, de l'ordre de celui de la génération 2004 (13\%), mais supérieur à celui de l'ensemble des diplômés bac + 4 (10\%). Cependant, l'importance du taux de chômage pour l'ensemble des diplômés de la culture est principa- lement imputable aux diplômés de la filière arts plastiques, pour lesquels il atteint $20 \%$. À l'opposé, les diplômés de l'architecture ont un taux de chômage très faible, de l'ordre de $4 \%$.

Pour $80 \%$ des chômeurs à la date de l'enquête, il s'agit d'un vrai décrochage de l'emploi, avec un chômage persistant ou récurrent. Enfin, un quart de l'ensemble des demandeurs d'emploi n'a jamais travaillé au cours des trois ans d'insertion.

\section{Correspondance entre le niveau d'emploi et le niveau de diplôme après trois ans d'activité}

Presque un emploi occupé sur deux est de niveau cadres et professions intellectuelles supérieures (tableau 9), soit $90 \%$ des emplois occupés par les diplômés de la culture, professions intermédiaires comprises, trois années après leur entrée sur le marché du travail. À titre de comparaison, c'est le cas pour $80 \%$ des diplômés de niveau bac +4 et pour $22 \%$ seulement de l'ensemble des emplois.

Le pourcentage de déclassés selon le critère du diplôme varie de $17 \%$ pour les diplômés de niveaux I et II du spectacle à $34 \%$ pour les diplômés de l'architecture. Mais si l'on croise les deux

Tableau 8 - Situation après trois années d'insertion professionnelle

\begin{tabular}{|c|c|c|c|c|c|c|c|}
\hline & Emploi & Chômage & Inactivité & Formation & $\begin{array}{l}\text { Reprise } \\
\text { d'études }\end{array}$ & Total & $\begin{array}{c}\text { Taux } \\
\text { de chômage }\end{array}$ \\
\hline Architecture & 93 & 4 & 2 & 0 & 1 & 100 & 4 \\
\hline Spectacle & 90 & 6 & 4 & - & - & 100 & 6 \\
\hline Arts plastiques & 71 & 18 & 5 & 4 & 2 & 100 & 20 \\
\hline Musées, patrimoine & 74 & 10 & 6 & 5 & 5 & 100 & 10 \\
\hline Ensemble & 81 & 12 & 4 & 2 & 1 & 100 & 13 \\
\hline Ensemble Génération 2004 & 77 & 13 & 3 & \multicolumn{2}{|c|}{7} & 100 & 10 \\
\hline
\end{tabular}

Source : Cereq/DEPS, Enquête génération 2004, ministère de la Culture et de la Communication, 2009

Tableau 9 - Niveau de l'emploi à la date de l'enquête

\begin{tabular}{|c|c|c|c|c|c|}
\hline $\begin{array}{l}\text { Domaine } \\
\text { du diplôme }\end{array}$ & $\begin{array}{l}\text { Chefs d'entreprise, cadres } \\
\text { et professions intellectuelles supérieures }\end{array}$ & $\begin{array}{l}\text { Professions } \\
\text { intermédiaires }\end{array}$ & Employés & Ouvriers & Ensemble \\
\hline Architecture & 66 & 33 & - & 1 & 100 \\
\hline Arts plastiques & 25 & 62 & 12 & 6 & 100 \\
\hline \multicolumn{6}{|l|}{ Spectacle } \\
\hline niveaux I et II & 83 & 13 & 4 & - & 100 \\
\hline niveau III & 73 & 19 & 4 & 3 & 100 \\
\hline Musées, patrimoine & 30 & 39 & 31 & - & \\
\hline Ensemble & 47 & 43 & 7 & 3 & 100 \\
\hline
\end{tabular}


critères «emploi en lien avec le diplôme» et « niveau de diplôme et niveau de l'emploi », une très forte proportion de diplômés de la culture ont un emploi totalement ajusté à leur formation trois ans après leur sortie du système éducatif. En effet, les emplois sans déclassement sont globalement des emplois en lien avec le diplôme. De ce fait, on observe ainsi, trois ans après leur entrée sur le marché du travail, un taux d'emploi parfaitement ajusté, variant entre environ $57 \%$ et $80 \%$ selon la filière.

\section{Stabilité de l'emploi trois ans \\ après l'obtention du diplôme : quatre diplômés sur dix sont en CDI et deux sur dix sont indépendants}

Six diplômés de la culture sur dix sont en CDI ou indépendants à la date de l'enquête, parmi les individus en activité (tableau 10). Le contrat à durée indéterminée et le statut de travailleur indépendant sont très répandus parmi les diplômés de l'architecture et ceux des arts plastiques (respectivement $80 \%$ et $66 \%$ ). À l'inverse, une forte proportion d'individus travaille en CDD parmi les diplômés du spectacle.

Si pour les diplômés de l'architecture, il s'agit principalement d'emploi à temps complet (93\%), c'est moins vrai pour les autres filières. Moins d'un diplômé sur deux du spectacle travaille à temps complet à la date de l'enquête. Directement lié à la quotité du temps de travail, le salaire moyen des diplômés de l'architecture est supérieur aux deux autres filières, ce qui reste vrai même si l'on se réfère uniquement aux diplômés à temps complet : l'écart reste conséquent entre les diplômés de l'architecture et ceux des autres filières.

\section{DES DIPLÔMES QUI FAVORISENT}

\section{LA MOBILITÉ PROFESSIONNELLE}

\section{Trois ans d'insertion :}

\section{un cycle marqué par une première année propice à l'intégration dans la vie active}

Ils ne sont que $6 \%$ à n'avoir jamais travaillé au cours des trois premières années de vie active (même niveau que l'ensemble de la génération 2004, mais plus élevé que pour les diplômés de niveau bac +3 ou plus, de l'ordre de $3 \%$ ). Au cours des trois premières années d'insertion, on observe une forte hausse du nombre d'individus ayant un emploi la première année, plus faible la deuxième année puis une stabilisation au cours de la troisième année (graphique 2).

Au cours de la première année, la part de diplômés ayant trouvé leur premier emploi passe de la moitié aux trois quarts. L'année suivante, cette proportion s'élève à $79 \%$, pour se stabiliser à $80 \%$ la troisième année. Parallèlement, la part des diplômés au chômage diminue progressivement pour se stabiliser autour de $12 \%$.

Les emplois sont plus stables après trois ans : le nombre d'emplois en CDI augmente, de même que le nombre d'indépendants (tableau 11).

Avec la stabilité, les premières années apportent aussi une meilleure rémunération: on observe une hausse importante du salaire médian qui peut s'expliquer notamment par l'augmentation du nombre d'emplois à temps complet (+ 5 points sur la période). Cependant, même lorsqu' on retire l'effet de l'augmentation de la part des emplois à temps complet, le phénomène se confirme avec une augmentation du salaire médian qui passe de 1300 euros à 1600 euros.

\section{Tableau 10 - Situation à la date de l'enquête}

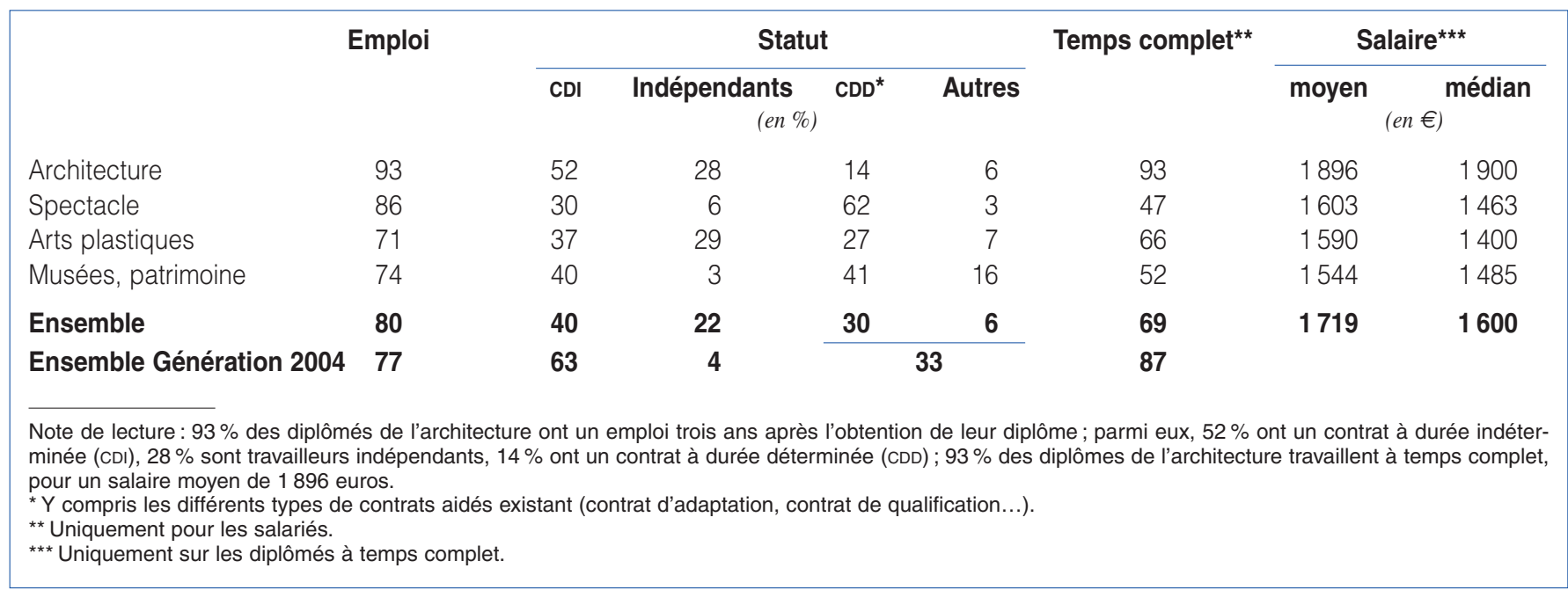




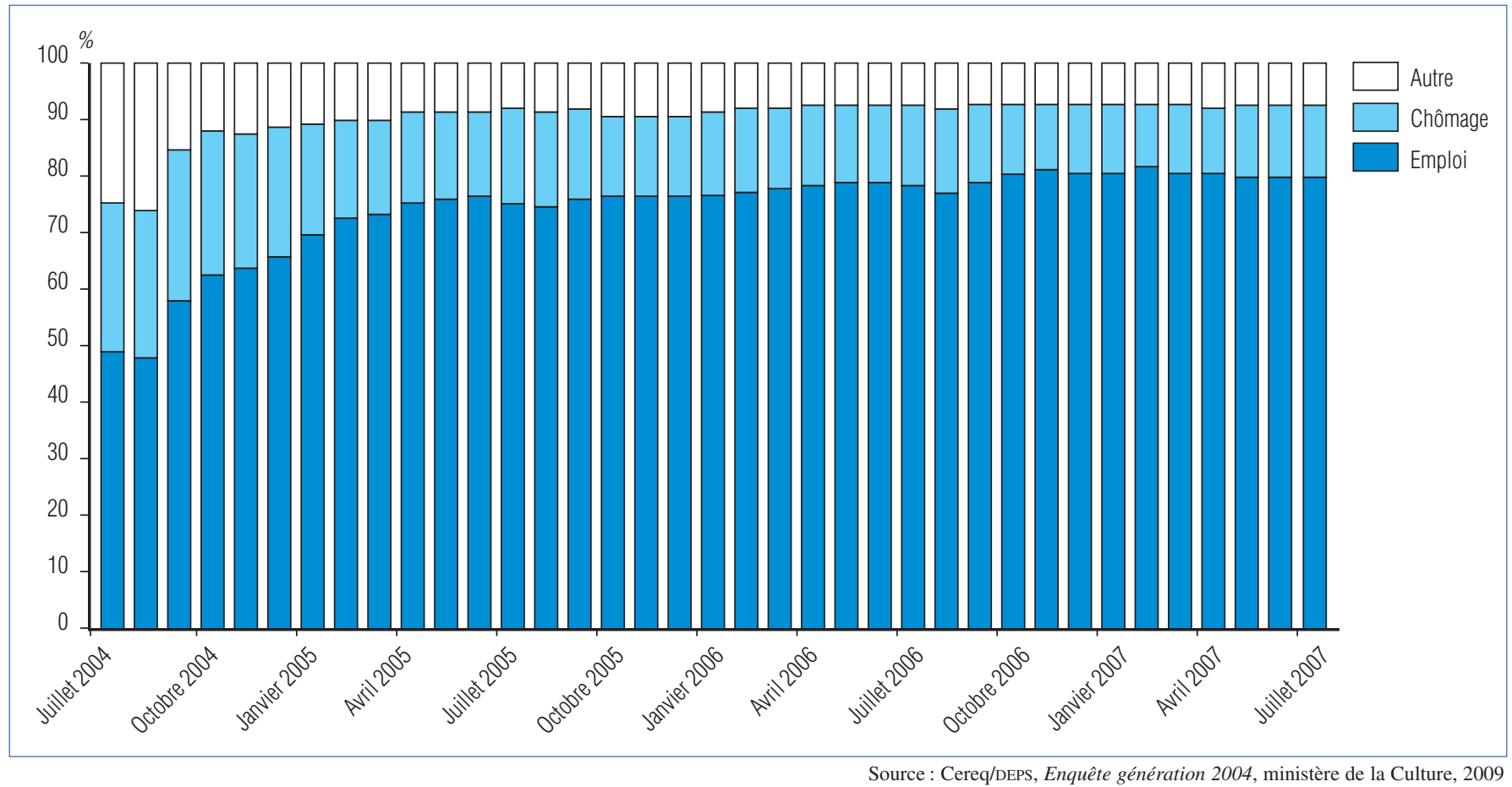

Tableau 11 - Évolution des contrats pour les individus ayant obtenu leur premier emploi en un an ou moins

\begin{tabular}{|c|c|c|}
\hline & $\begin{array}{l}\text { Premier } \\
\text { emploi* }\end{array}$ & $\begin{array}{c}\text { Emploi à la } \\
\text { date de l'enquête }\end{array}$ \\
\hline CDI & 23 & 43 \\
\hline CDD & 56 & 27 \\
\hline Contrats aidés & 2 & 2 \\
\hline Indépendants & 15 & 22 \\
\hline Autres & 4 & 5 \\
\hline Chômage & - & - \\
\hline Inactif & - & - \\
\hline Ensemble & 100 & 100 \\
\hline Proportion de temps complet & 65 & 70 \\
\hline Salaire net mensuel médian & $1200 €$ & $1500 €$ \\
\hline Ensemble Génération 2004 & $1100 €$ & $1300 €$ \\
\hline \multicolumn{3}{|c|}{$\begin{array}{l}\text { Note de lecture : } 23 \text { \% des premiers contrats des diplômés de la culture sont } \\
\text { des CDI. } \\
{ }^{*} \text { Il s'agit du premier emploi des diplômés qui l'ont eu en un an ou moins. } \\
{ }^{*} \text { Hors chômage et inactivité. }\end{array}$} \\
\hline
\end{tabular}

\section{La moitié des diplômés ont connu un} changement de situation au cours des trois premières années d'insertion

Entrer sur le marché de l'emploi avec un contrat à durée déterminée n'est pas pénalisant pour la suite de la carrière. On observe que plus de la moitié des individus ayant commencé en CDD ont connu un changement de statut, passant en CDI pour les deux
Tableau 12 - Changement de situation contractuelle

\begin{tabular}{|c|c|c|c|c|c|c|}
\hline \multirow{2}{*}{\multicolumn{2}{|c|}{$\begin{array}{l}\text { Changement } \\
\text { de situation* }\end{array}$}} & \multicolumn{5}{|c|}{ dont } \\
\hline & & $C D I$ & $C D D$ & $\begin{array}{l}\text { indé- } \\
\text { pen- } \\
\text { dants }\end{array}$ & $\begin{array}{l}\text { contrats } \\
\text { aidés }\end{array}$ & autres \\
\hline CDI & 20 & - & 7 & 9 & 0 & 4 \\
\hline CDD & 55 & 38 & - & 11 & 2 & 4 \\
\hline Indépendants & 14 & 11 & 1 & - & 0 & 2 \\
\hline Contrats aidés & 44 & 11 & 28 & 6 & - & 0 \\
\hline Ensemble & 39 & & & & & \\
\hline \multicolumn{7}{|c|}{$\begin{array}{l}\text { Note de lecture : } 20 \% \text { des diplômés ayant commencé en CDI ont connu un } \\
\text { changement de situation (en termes de contrat). } \\
{ }^{*} \text { Hors sortie pour chômage, formation, inactivité... }\end{array}$} \\
\hline
\end{tabular}

tiers d'entre eux. Une petite partie des individus en CDI lors de la première année sont aujourd'hui indépendants (environ $10 \%$, voir tableau 12). Enfin, Les indépendants restent fortement indépendants. Seuls $14 \%$ d'entre eux ont changé de statuts, se trouvant pour la plupart en CDI à la date de l'enquête.

\section{Les mobilités dans l'emploi}

Presque un diplômé de la culture sur deux n'a connu qu'un seul employeur (y compris lui-même dans le cas de travailleurs indépendants), près d'un tiers a changé une fois et un peu moins d'un quart plus de deux fois. De ce fait, les diplômés de la 
culture ont connu une relative stabilité : en moyenne plus de 19 mois par employeur (tableau 13). Les diplômés du spectacle sont, parmi les diplômés de la culture, ceux qui ont été les plus stables au cours de leurs trois premières années, les deux tiers d'entre eux n'ayant connu qu'un employeur au cours des trois premières années.

\section{Tableau 13 - Mobilités au cours des trois premières années d'insertion}

\begin{tabular}{|c|c|c|c|c|c|}
\hline & \multicolumn{4}{|c|}{$\begin{array}{c}\text { Nombre d'employeurs } \\
\text { depuis l'obtention } \\
\text { du diplôme* }\end{array}$} & \multirow[t]{2}{*}{$\begin{array}{c}\text { Temps } \\
\text { moyen } \\
\text { par employeur }\end{array}$} \\
\hline & Un & Deux & $\begin{array}{l}\text { Trois } \\
\text { u plus }\end{array}$ & Total & \\
\hline \multirow[b]{2}{*}{ Architecture } & \multicolumn{4}{|c|}{$(e n \%)$} & (en mois) \\
\hline & 43 & 37 & 20 & 100 & 20,5 \\
\hline Spectacle & 67 & 17 & 16 & 100 & 22,5 \\
\hline Arts plastiques & 46 & 30 & 24 & 100 & 17,2 \\
\hline $\begin{array}{l}\text { Musées, } \\
\text { patrimoine }\end{array}$ & 44 & 45 & 11 & 100 & 16,1 \\
\hline Ensemble & 50 & 29 & 21 & 100 & 19,2 \\
\hline $\begin{array}{l}\text { Note de lecture : } 43 \\
\text { employeur au cour } \\
\text { * Sur l'ensemble de } \\
\text { vacances. }\end{array}$ & $\begin{array}{l}\% \text { de } \\
\text { s de I } \\
\text { s dipl }\end{array}$ & $\begin{array}{l}\text { diplômé } \\
\text { rss trois } \\
\text { nés aya }\end{array}$ & $\begin{array}{l}\text { de l'archit } \\
\text { emières } \\
\text { eu au mo }\end{array}$ & $\begin{array}{l}\text { tecture r } \\
\text { années } \\
\text { ins un } €\end{array}$ & $\begin{array}{l}\text { ont connu qu'un seul } \\
\text { insertion. } \\
\text { iploi, hors emploi de }\end{array}$ \\
\hline
\end{tabular}

Source : Cereq/DEPS, Enquête génération 2004, ministère de la Culture et de la Communication, 2009
Plus du quart des diplômés de la culture ayant un emploi trois ans après leur insertion sont à la recherche d'un nouvel emploi

Bien qu'ils aient un emploi à la date de l'enquête, une partie des diplômés ( $28 \%$ ) recherche un autre emploi, soit une proportion légèrement supérieure à celle de l'ensemble de la génération 2004 (23\% sont dans ce cas). C'est essentiellement le cas pour les personnes en CDD ou en contrats aidés (contrat d'adaptation, contrat de qualification...) qui sont $45 \%$ à chercher un nouvel emploi. Si seulement $15 \%$ des diplômés de l'architecture sont dans ce cas, environ un tiers des diplômés des autres filières se déclarent à la recherche d'un nouvel emploi. Cette différence entre les diplômés de l'architecture et les autres s'explique par la forte part de contrats à durée indéterminée et de travail indépendant dans cette branche. Enfin, la proportion des diplômés de la filière arts plastiques à occuper un emploi sans lien avec leur diplôme explique aussi ce désir de changer d'emploi. 


\section{Typologie des emplois des jeunes diplômés de la culture : quatre trajectoires types d'insertion}

Globalement, les diplômés de la culture ont suivi principalement quatre types de trajectoire* :

$-61 \%$ d'entre eux ont connu un accès rapide et durable à l'emploi (que celui-ci soit en rapport ou non avec leur filière) ;

$-16 \%$ ont eu un accès différé à l'emploi, que ce soit après une période de chômage, de formation ou d'inactivité (tableau 14).

$-8 \%$ ont connu un chômage persistant ou récurrent ;

$-7 \%$ ont connu un décrochage de l'emploi.

Ces propositions sont d'une façon générale conformes à l'ensemble de la génération 2004.

Selon la filière, on constate quelques différences importantes. $82 \%$ des diplômés de l'architecture connaissent ainsi entre 2004 et 2007 un accès rapide et durable à l'emploi, alors que ce n'est le cas que pour 46 \% des diplômés 2004 des arts plastiques.

\section{Tableau 14 - Description du parcours d'insertion}

\begin{tabular}{|c|c|c|c|c|c|c|}
\hline & rchitecture & $\begin{array}{c}\text { Arts } \\
\text { plastiques }\end{array}$ & Spectacle & $\begin{array}{l}\text { Musées, } \\
\text { patrimoine }\end{array}$ & $\begin{array}{l}\text { Ensemble } \\
\text { des diplômés }\end{array}$ & $\begin{array}{l}\text { Ensemble } \\
\text { Génération } 2004\end{array}$ \\
\hline $\begin{array}{l}\text { Accès rapide et durable à l'emploi } \\
\text { Accès différé à l'emploi }\end{array}$ & 82 & 45 & 78 & 53 & 61 & 58 \\
\hline $\begin{array}{l}\text { après une période de chômage } \\
\text { Accès différé à l'emploi après }\end{array}$ & 5 & 16 & 6 & 10 & 11 & 12 \\
\hline une période d'inactivité ou de formation & 4 & 5 & 4 & 8 & 5 & 4 \\
\hline Chômage persistant ou récurrent & 2 & 12 & 6 & 7 & 8 & 8 \\
\hline Décrochage de l'emploi & 4 & 10 & 2 & 6 & 7 & 8 \\
\hline Inactivité durable & 1 & 6 & 1 & 9 & 4 & 3 \\
\hline Formation ou reprise d'études de longue durée & 1 & 2 & 1 & 4 & 2 & 4 \\
\hline Formation ou reprise d'études de courte durée & 1 & 4 & 2 & 4 & 3 & 3 \\
\hline Ensemble & 100 & 100 & 100 & 100 & 100 & \\
\hline
\end{tabular}

Note de lecture : $82 \%$ des diplômés de l'architecture ont eu un accès durable et rapide à l'emploi au cours de leurs trois premières années d'insertion.

Source : Cereq/DEPS, Enquête génération 2004, ministère de la Culture et de la Communication, 2009

* Ces typologies de parcours ont été élaborées selon la méthode du Laboratoire interdisciplinaire de recherche sur les ressources humaines et l'emploi (Lirhe) afin de prendre en compte l'intégralité du parcours professionnel des jeunes, au cours des trois années écoulées entre la fin des études et la date d'interrogation. Les trajectoires suivies par chaque jeune ont ainsi été regroupées en fonction de leur proximité : deux jeunes ont des trajectoires d'autant plus proches qu'ils traversent au même moment la même situation ; inversement, ils ont des trajectoires éloignées lorsque le nombre de mois où leur situation diffère est élevé. Neuf trajectoires types ont ainsi été identifiées, regroupées en quatre groupes.

\section{Présentation de l'enquête Génération 2004}

À la suite des enquêtes Génération 1992, 1998 et 2001, le Cereq a interrogé en 2007 un échantillon des 37000 personnes issues de formation initiale en 2004. Cette année-là, environ 705000 personnes sont sorties diplômées du système éducatif, 65000 ont été interrogées. À chaque enquête, le Cereq permet à des collectivités publiques (ministères ou collectivités territoriales) d'étendre le champ de l'enquête à une zone géographique ou à un domaine de formation particuliers. À la demande du ministère de la Culture et de la Communication, une extension à l'enquête nationale a donc été réalisée en direction des diplômés des filières culturelles et artistiques et a concerné 1270 individus.

L'objectif de cette enquête inédite est d'observer l'insertion professionnelle des diplômés des établissements d'enseignement supérieur relevant du ministère de la Culture et de la Communication : elle décrit leur situation mois par mois les trois premières années après l'obtention de leur diplôme en 2004. L'enquête concerne les diplômés des filières suivantes : architecture (uniquement les DPLG), arts plastiques, musées et patrimoine, musique, danse, théâtre, spectacle et cinéma*.

*Voir «L'enseignement supérieur Culture», document réalisé par les services du ministère de la Culture et de la Communication, disponible sur www.culture.gouv.fr/culture/infos-pratiques/rubrique formation. 


\section{RÉSUMÉ}

L'enquête sur l'insertion professionnelle des 5500 diplômés d'établissements supérieurs relevant du ministère de la Culture et de la Communication au cours des trois années qui suivent l'obtention de leur diplôme en 2004 révèle la qualité de ces formations. Professionnalisantes à court terme, elles garantissent un accès rapide à un emploi en lien avec la formation reçue pour près de quatre diplômés sur cinq. Les résultats confirment l'importance, pour la pérennité de l'emploi, de la correspondance entre le domaine et le niveau de formation et le premier emploi exercé. Trois ans après l'obtention de leur diplôme, $81 \%$ des diplômés de la culture travaillent, contre $77 \%$ de l'ensemble de la génération diplômée en 2004. Enfin, la rapidité de l'insertion, le niveau de rémunération et le taux de chômage varient selon les filières : l'architecture insère rapidement ses diplômés qui travaillent le plus souvent à temps complet, tandis que les diplômés du spectacle travaillent plus souvent à temps partiel et ceux des arts plastiques connaissent un taux de chômage de 20 \%. L'enquête, menée en collaboration avec le Centre d'études et de recherches sur les qualifications (Cereq) auprès de 120 établissements et concernant plus de 1200 diplômés, est inédite pour les diplômés de la culture.

\section{ABSTRACT}

The survey of the professional integration of 5,500 graduates from higher education establishments coming under the Ministry of Culture and Communication in the three years following obtaining their degree in 2004 reveals the quality of these courses. Providing immediately useable professional training, they guarantee quick access to employment related to the training received for nearly four graduates out of five. The results confirm the importance, for continuity in employment, of a match between the field, the level of education and the first job undertaken. Three years after obtaining their degree, $81 \%$ of culture graduates are working, against $77 \%$ of the whole generation graduating in 2004. Finally, the speed of integration, the remuneration level and the unemployment rate vary with the courses followed: architecture inserts its graduates, who most often work full time, quickly, whereas entertainment graduates most often work part time and those in the plastic arts experience an $20 \%$ unemployment rate. The survey, carried out in collaboration with the Centre for Study and Research on Qualifications (Cereq), included 120 establishments and covered 1,200 graduates and is unprecedented for graduates in cultural subjects..

\section{L'essentiel en quelques chiffres}

- 5500 diplômés d'un établissement supérieur artistique et culturel relevant du ministère de la Culture et de la Communication.

- $\mathbf{8 0} \%$ des diplômés obtiennent un emploi en lien avec leur domaine de formation.

- 75 \% des diplômés trouvent leur emploi au cours de la première année qui suit l'obtention du diplôme.

- 20 mois : durée moyenne du premier emploi.

- $65 \%$ des emplois sont à temps complet : $91 \%$ pour la filière architecture, $43 \%$ pour la filière spectacle.

- $\mathbf{8 1} \%$ des diplômés travaillent trois ans après l'obtention de leur diplôme.

- $40 \%$ sont en CDI, $30 \%$ sont en CDD, $22 \%$ ont un statut de travailleur indépendant. 


\section{Les dépenses culturelles des collectivités locales en 2006 : près de 7 milliards d'euros pour la culture}

\section{Jean-Cédric Delvainquière, Bruno Dietsch}

Depuis 1978, les enquêtes statistiques nationales relatives aux dépenses culturelles des collectivités locales manifestent leur montée en puissance et démontrent le développement de la décentralisation culturelle ainsi que l'implication réelle des collectivités dans la vie culturelle. Les résultats de l'année budgétaire 2006 mettent particulièrement en évidence l'importance de l'échelon intercommunal.

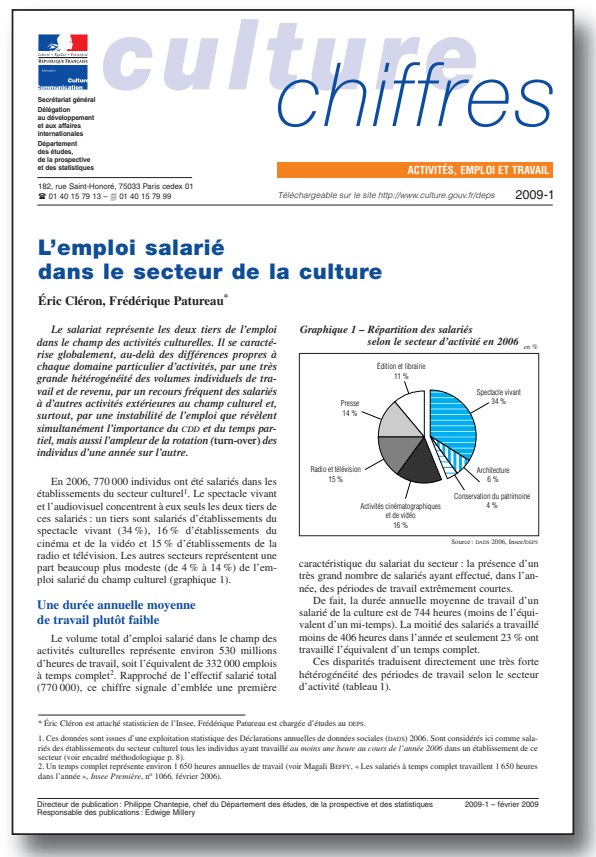

\section{L'emploi salarié} dans le secteur de la culture

\section{Éric Cléron, Frédérique Patureau}

Le salariat représente les deux tiers de l'emploi dans le champ des activités culturelles. Il se caractérise globalement, au-delà des différences propres à chaque domaine particulier d'activités, par une très grande hétérogénéité des volumes individuels de travail et de revenu, par un recours fréquent des salariés à d'autres activités extérieures au champ culturel et, surtout, par une instabilité de l'emploi que révèlent simultanément l'importance du CDD et du temps partiel, mais aussi l'ampleur de la rotation (turn-over) des individus d'une année sur l'autre.

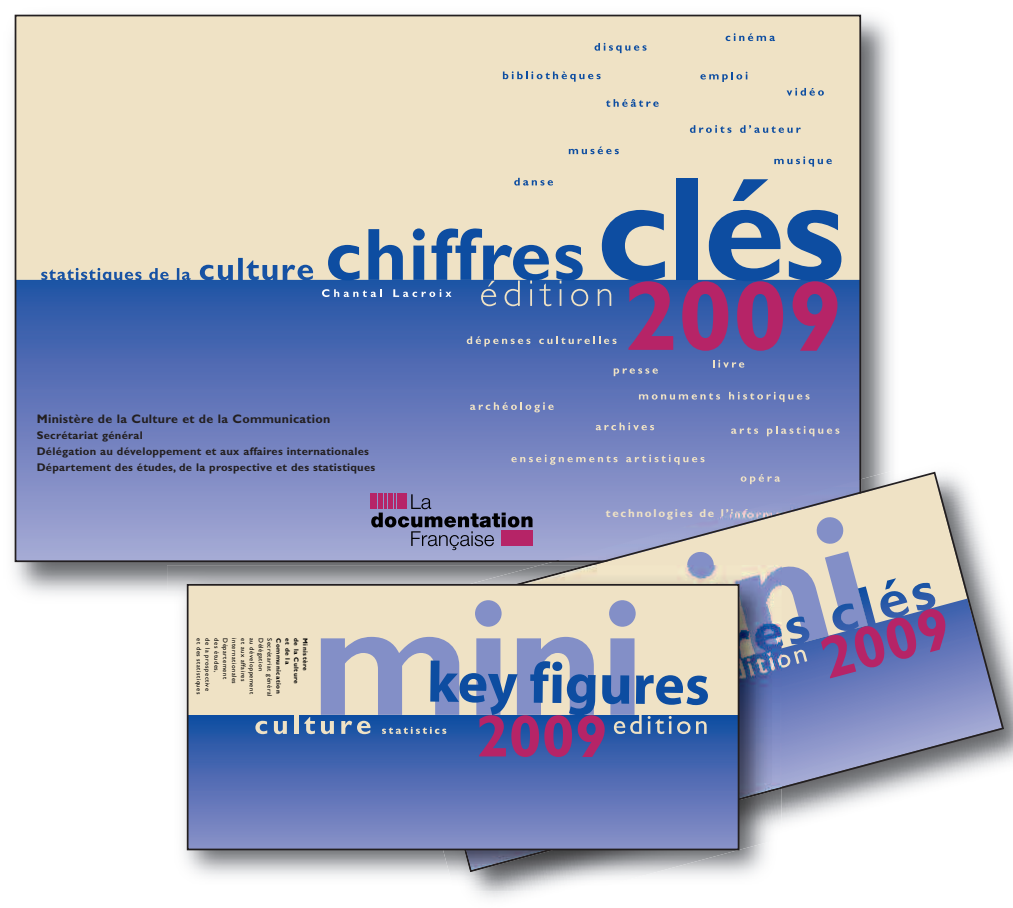

\section{Chiffres clés,} statistiques de la culture

Présentation sous forme de tableaux et graphiques des statistiques relatives à la vie culturelle en France. L'étude aborde le spectre très large du champ culturel (patrimoine, spectacle, enseignements artistiques...) et propose une approche transversale sur les grands thèmes de politique culturelle (emploi, financement, pratiques). Véritable outil de travail pour tous ceux - professionnels, responsables politiques et administratifs qui souhaitent éclairer leur action et la situer dans le contexte national, il constitue un état de la culture annuel qui fait désormais modèle. Une version abrégée, Mini chiffres clés, est également disponible en français et en anglais.

L'édition 2009 est publiée sous deux formes: imprimée (en vente à la Documentation française) et téléchargeable par secteur sur le site du ministère de la Culture et de la Communication.

Tous les documents publiés par le DEPS sont téléchargeables sur http://www.culture.gouv.fr/deps

Le DEPS n'assurant pas de diffusion physique de ces documents, nous vous proposons de vous informer régulièrement des parutions par message électronique. Pour ce faire, merci de bien vouloir nous communiquer votre courriel à l'adresse contactdeps.ddai@culture.gouv.fr en indiquant comme sujet du message : «diffusion des collections du Deps ». 